Arq. Bras. Med. Vet. Zootec., v.61, n.4, p.968-979, 2009

\title{
Associação entre medidas lineares e angulares de equinos da raça Mangalarga Marchador
}

\author{
[Phenotypic association between linear and joint angle traits of Mangalarga Marchador horses] \\ M.C.G.R. Lage $^{1}$, J.A.G. Bergmann ${ }^{2}$, A.M. Procópio ${ }^{3}$, J.C.C. Pereira ${ }^{2}$, J. Biondini ${ }^{2}$ \\ ${ }^{1}$ Pontifícia Universidade Católica de Minas Gerais \\ Rua do Rosário, 1081 \\ 32630-000 - Betim, MG \\ ${ }^{2}$ Escola de Veterinária - UFMG - Belo Horizonte, MG \\ ${ }^{3}$ Faculdade de Estudos Administrativos de Minas - Belo Horizonte, MG
}

\begin{abstract}
RESUMO
Foram analisadas 12 medidas de comprimento dos ossos, duas medidas de altura, duas medidas de perímetro e 11 medidas de ângulos articulares) de 169 equinos da raça Mangalarga Marchador, de ambos os sexos (82\% de fêmeas), com idades entre 35 e 269 meses, de 11 criatórios do Estado de Minas Gerais e cinco de São Paulo. Os efeitos de estado e criatório de origem, tipo de criação (baia ou pasto), idade e sexo sobre essas características foram avaliados pelo método dos quadrados mínimos, e as associações entre as características foram quantificadas por meio da correlação de resíduos. Os modelos justificaram pouco da variação observada, com coeficientes de determinação variando de 0,09 a 0,48 para as medidas lineares e de 0,11 a 0,44 para as medidas angulares. Estado e criatório de origem, sexo, idade e tipo de criação foram importantes fontes de variação, respectivamente para $13(48,1 \%), 12(44,4 \%)$, seis $(22,2 \%)$, quatro $(14,8 \%)$ e três $(11,1 \%)$ das 27 características avaliadas. As correlações de resíduo entre as características indicaram que a escolha de animais com úmero de maior comprimento está associada a animais com membros torácicos e pélvicos mais longos e que a escolha de animais a partir do tamanho dos membros acarretará concomitante incremento na altura da cernelha e na garupa e do perímetro da canela e torácico. Ainda, a escolha dos animais considerando as medidas dos ângulos metacarpofalangeano e dedo torácico com a horizontal refletirá positivamente na altura da cernelha e da garupa.
\end{abstract}

Palavras-chave: equino, morfometria, medidas lineares, medidas angulares

\begin{abstract}
The effects of State and stud of origin, management (on pasture or in stable), gender, and age on morphometrics traits (measurements of 12 bone segments, 11 joint angles, two perimeters, and two heights) of 169 Mangalarga Marchador horses (82\% females) raised in 11 herds of Minas Gerais and five from São Paulo State, were evaluated using the least squares methodology. In addition, residual phenotypic associations among these traits were measured. Statistical models did not fit well the observed variation, with coefficients of determination varying from 0.09 to 0.48 for linear and from 0.11 to 0.44 for angular traits. State and stud of origin, gender, age, and management were important sources of variation, respectively on 13 (48.1\%), 12 (44.4\%), six (22.2\%), four (11.1\%), and three (11.1\%) of the 27 evaluated traits. Residual correlations between traits indicated that selection of animals with longer humerus was associated to animals with longer front and rear leg bone segments and higher wither and croup heights and larger crop and cannon perimeters. Also, selection of animals with larger fetlock and coronet-horizontal line angles will positively reflect on the wither and croup heights of the animals.
\end{abstract}

Keywords: equine, morphometrics, linear traits, angle traits

Recebido em 27 de novembro de 2008

Aceito em 9 de julho de 2009

E-mail: coeli@pucminas.br 


\section{INTRODUÇÃO}

No Brasil, a raça equina Mangalarga Marchador é a que apresenta o maior número de animais registrados e de registros anuais (Costa et al., 2004). A raça, considerada de sela e que apresenta o andamento marchado como principal característica funcional, tem sido amplamente utilizada para o trabalho no campo, para o lazer e em competições equestres. Sabe-se que o conhecimento dos fatores que influenciam as medidas morfológicas e das associações entre estas e outros atributos funcionais é importante para a definição do tipo de treinamento e para a seleção dos equinos marchadores (Nascimento, 1999).

A mensuração das diversas partes anatômicas do animal como critério para a avaliação da conformação dos equinos tem longa história. Já no início do século XV, Leonardo da Vinci tentou descrever objetivamente a conformação do cavalo. Da mesma forma, Claude Bourgelat, no século XVIII, examinou diversas mensurações, expressando-as em relação ao comprimento da cabeça. No início do século passado, Simon von Nathusis avaliou a morfometria dos equinos de forma objetiva (Mota, 1999). Desde então, tem sido constante a busca por dispositivos e técnicas que possibilitem a correta, prática e econômica quantificação das dimensões das diversas partes anatômicas e zootécnicas dos equinos.

Mota (1999) afirmou que no clássico trabalho de Goubaux e Barrier (1884) L'extérieur de Cheval, discutiu-se a possibilidade de mensurar os ângulos das articulações dos cavalos com base em fotografias, tiradas de perfil. De acordo com esse autor, a vantagem seria que todos os ângulos são registrados simultaneamente, ao contrário de registros tomados diretamente nos animais. Entretanto, para aumentar a confiabilidade das mensurações do cavalo, quando se empregam métodos tradicionais, com o uso de hipômetro, fita métrica ou trena, Hickman e Colles (1984) desenvolveram esquema piloto de mensuração, utilizando feixe de laser, obtendo confiança de $1 \mathrm{~mm}$ nas medidas de altura na cernelha. Mais recentemente, Procópio et al. (2007) utilizaram a cinematografia e imagens digitalizadas para caracterizar a variabilidade das medidas angulares dos equinos marchadores brasileiros durante a locomoção, por meio das curvas de ângulo-tempo, correlacionando-as com a fase de locomoção do animal e comparando-as com as dos cavalos de sela europeus.

Apesar das várias iniciativas para a caracterização morfológica das raças equinas criadas no Brasil (Costa et al., 1998; Mota, 1999; Procópio et al., 2007), não foram encontrados na literatura disponível estudos que avaliassem as associações entre medidas morfométricas lineares e angulares de equinos marchadores.

Os objetivos deste estudo foram determinar o comprimento de diferentes ossos longos, ângulos articulares e perimetrias torácica e metacarpiana, com possíveis associações fenotípicas, e avaliar a importância dos efeitos dos fatores ambiente, sexo e idade sobre essas características de animais adultos da raça Mangalarga Marchador.

\section{MATERIAL E MÉTODOS}

Foram utilizadas informações de 169 animais da raça Mangalarga Marchador, de ambos os sexos, $82 \%$ de fêmeas, criados em pasto ou em baias, com média de idade de 91,7 $\pm 46,2$ meses, nascidos e criados em 11 criatórios do Estado de Minas Gerais e cinco do Estado de São Paulo.

Doze medidas de comprimento dos ossos, duas de perímetro e duas de altura, e 11 medidas de ângulos articulares dos membros torácicos e pélvicos foram obtidas, unilateralmente, por um técnico treinado e credenciado pela Associação Brasileira dos Criadores do Cavalo Mangalarga Marchador, segundo normas e exigências do Registro Genealógico da Associação (Estatuto..., 1992).

As medidas lineares avaliadas foram: alturas na cernelha e na garupa; perímetros torácico e da região do metacarpo (canela anterior); e comprimentos da escápula, do úmero (braço), do rádio, da canela, do metacarpo ( $3^{\circ}$ metacarpiano), das quartelas (falange proximal ou primeira falange) torácica e pelvica, da garupa (coxal), do fêmur, da tíbia, do tarso (tuberosidade calcânea ou jarrete), e do metatarso (terceiro metatarsiano ou canela posterior). No membro torácico, as medidas angulares foram: escápula com horizontal, escapuloumeral, umerorradioulnar (umerorradial), metacarpofalangeano e dedo com a horizontal. No membro pélvico, as medidas angulares foram: coxal com horizontal (ângulo 
axial coxal ou coxal), coxofemoral, femorotibial, tibiotarsicometatarsico, metatarsofalangeano (metatarsofalange) e dedo com a horizontal. A completa descrição e a nominação anatômica e zootécnica das características avaliadas foram feitas por Lage (2004).

As mensurações foram realizadas nos criatórios de origem, com os animais colocados em local tranquilo, de piso regular e plano, e posicionados com os membros torácicos paralelos. As porções anteriores dos cascos ficavam em linha, com diferença máxima de $1,5 \mathrm{~cm}$. Manteve-se a direção cranial dos cascos dos membros pélvicos em relação aos torácicos, com diferença máxima de $15 \mathrm{~cm}$. A cabeça foi mantida em posição natural em relação ao pescoço, e foi tomada como referência a linha imaginária horizontal, entre o olho e o ponto mais alto da cernelha. Para sinalizar os pontos anatômicos de referência para as mensurações angulares, foi utilizado papel adesivo com $0,5 \mathrm{~cm}$ de diâmetro, colocado o mais próximo dos centros geométricos de rotação das articulações (Lage, 2004). Para as medidas de comprimento, foram delimitadas as extremidades dos ossos visíveis durante a locomoção, segundo Leach et al. (1984).

As medidas lineares foram obtidas com hipômetro metálico e fita métrica convencional. Para as medidas angulares, obtidas com artrogoniômetro, foram adotados os pontos de referência anatômica (Lage, 2004, Procópio et al., 2007). O artrogoniômetro utilizado constou de um compasso com hastes longas, uma das quais é fixa e munida de fio de prumo e nível e a outra móvel, que gira em torno do transferidor (Lazzeri, 1992). Para a mensuração dos ângulos, colocou-se o centro da articulação do compasso sobre o centro de movimento do ângulo, com os ramos do compasso posicionados sobre o eixo dos raios ósseos, e procedeu-se à leitura no círculo graduado.

Os procedimentos para organização dos arquivos, cálculos de variáveis, análises preliminares e análises estatísticas foram realizados utilizando subrotinas incluídas no pacote estatístico SAS/1990. Estatísticas descritivas, frequências, médias, desvio-padrão, valores máximos e mínimos e coeficientes de variação foram calculados para todas as variáveis. As características lineares e angulares foram submetidas à modelagem pelo método dos quadrados mínimos para avaliar a importância dos efeitos das variáveis fixas independentes: Estado de origem (São Paulo ou Minas Gerais), criatório aninhado dentro de Estado (cinco criatórios de São Paulo e 11 de Minas Gerais), tipo de criação (em baia ou em pasto), e sexo e idade do animal como covariável. O nível de significância adotado para avaliar os efeitos desses fatores foi de $5 \%$.

No intuito de avaliar as associações entre as características, foram obtidas correlações de Pearson a partir dos valores residuais oriundos dos modelos acima definidos.

\section{RESULTADOS E DISCUSSÃO}

As características lineares e angulares, com as respectivas médias e desvios-padrão, coeficientes de variação, valores máximos e mínimos estão apresentados na Tab. 1. As mensurações da altura na cernelha e na garupa, comprimento da escápula e perímetro da canela e do tórax foram as únicas encontradas com descrição na literatura, em trabalhos realizados com a raça Mangalarga Marchador (Carneiro et al., 1952; Barbosa, 1993; Zamborlini, 1996; Zamborlini et al., 1996). As alturas na cernelha e na garupa observadas no presente estudo foram próximas às apresentadas por outros autores, em plantéis e épocas distintas (Jordão e Camargo, 1950; Barbosa, 1993; Zamborlini, 1996).

As menores variações das medidas lineares foram obtidas para a altura na cernelha $(1,8 \%)$ e na garupa $(1,8 \%)$. Para as medidas lineares restantes, o coeficiente de variação variou de $3,1 \%$, para o comprimento coxal, a $14,5 \%$, para o comprimento da tíbia. As menores variações das duas medidas de altura retratam a uniformidade dos animais Mangalarga Marchador deste estudo, principalmente por serem de animais adultos. Além disso, evidenciam a maior facilidade para a obtenção dessas medidas. A maior variação no comprimento da tíbia reflete as limitações para sua obtenção devido à sua localização anatômica, que exige adequado e seguro posicionamento do técnico. A dificuldade em manter o animal estático, sem reação ao contato com o hipômetro, pode ter contribuído para a maior variação dessa característica. 
Tabela 1. Médias observadas, desvios-padrão (DP), coeficientes de variação (CV) e valores mínimos (Mín.) e máximos (Máx.) das medidas lineares e angulares de equinos da raça Mangalarga Marchador

\begin{tabular}{lccccc}
\hline \multicolumn{1}{c}{ Característica } & Média & DP & CV, \% & Mín & Máx \\
\hline Linear (cm) & & & & & \\
\hline Comprimento da escápula & 60,0 & 1,9 & 3,23 & 53,0 & 66,0 \\
Comprimento do úmero & 34,0 & 1,2 & 3,63 & 30,0 & 39,0 \\
Comprimento do rádio & 35,0 & 1,8 & 5,02 & 26,0 & 40,0 \\
Comprimento da canela & 9,0 & 0,7 & 7,74 & 7,0 & 11,0 \\
Comprimento do metacarpo & 22,0 & 1,4 & 6,32 & 16,0 & 30,0 \\
Comprimento da quartela torácica & 11,0 & 0,7 & 5,93 & 8,7 & 13,0 \\
Comprimento da garupa ou coxal & 54,0 & 1,7 & 3,09 & 47,0 & 59,0 \\
Comprimento do fêmur & 35,0 & 2,0 & 5,83 & 29,0 & 40,0 \\
Comprimento da tíbia & 31,0 & 4,5 & 14,50 & 25,0 & 85,0 \\
Comprimento do tarso & 10,0 & 0,9 & 8,70 & 7,0 & 13,0 \\
Comprimento do metatarso & 27,0 & 1,7 & 6,39 & 20,0 & 38,0 \\
Comprimento da quartela pélvica & 11,0 & 1,0 & 8,90 & 9,0 & 21,0 \\
Altura na cernelha & 149,0 & 2,7 & 1.81 & 140,0 & 156,0 \\
Altura na garupa & 147,7 & 2,6 & 1,78 & 139,0 & 155,0 \\
Perímetro da canela & 17,0 & 0,6 & 3,61 & 16,0 & 20,0 \\
Perímetro torácico & 177,0 & 6,1 & 3,42 & 160,0 & 192,0 \\
\hline Angular (graus) & & & & & \\
\hline Escápula com horizontal & 62,4 & 2,9 & 4,48 & 52,0 & 70,0 \\
Escapuloumeral & 88,1 & 6,5 & 6,39 & 80,0 & 135,0 \\
Umerorradial & 130,7 & 5,5 & 4,17 & 118,0 & 155,0 \\
Metacarpofalange & 145,0 & 6,6 & 4,12 & 112,0 & 159,0 \\
Dedo torácico com a horizontal & 54,5 & 5,2 & 9,13 & 40,0 & 69,0 \\
Coxal & 53,8 & 4,8 & 7,07 & 40,0 & 66,0 \\
Coxofemoral & 138,0 & 6,8 & 4,28 & 120,0 & 161,0 \\
Femorotibial & 151,0 & 5,1 & 3,16 & 131,0 & 166,0 \\
Tibiotarsicometatársico & 154,4 & 5,7 & 3,57 & 105,0 & 164,0 \\
Metatarsofalange & 153,1 & 10,0 & 6,52 & 60,0 & 171,0 \\
Dedo pélvico com a horizontal & 57,9 & 5,7 & 9,04 & 43,0 & 70,0 \\
\hline
\end{tabular}

Para as medidas angulares, as variações foram próximas às das medidas lineares, sendo a medida do ângulo femorotibial a de menor coeficiente de variação (3,2\%), e a do ângulo do dedo torácico com a horizontal a de maior coeficiente de variação $(9,1 \%)$. O resultado evidencia dificuldades para a identificação dos pontos anatômicos de referência para a obtenção das medidas angulares, relacionadas à grande influência do posicionamento do animal, da sua cobertura muscular, da elasticidade da pele e da capacidade em permanecer em estática. Aliado a isso, os animais apresentaram maior rejeição ao contato com o artrogoniômetro do que ao hipômetro, pois esses animais são mais frequentemente expostos às mensurações das características lineares do que às angulares.

Com base nos de modelos estatísticos que incluíram os efeitos fixos de estado, criatório aninhado dentro de estado, tipo de criação, sexo e idade do animal, as médias das medidas lineares e angulares foram ajustadas pelo método dos quadrados mínimos (Tab. 2). Os valores dos coeficientes de determinação para os modelos que incluíram medidas lineares como variáveis dependentes variaram de 0,09 , para o comprimento do rádio, a 0,48 , para $\mathrm{o}$ comprimento do tarso. Já para variáveis angulares, a variação dessa estatística foi de 0,11 a 0,44, respectivamente, para os ângulos metatarsofalangeano e do coxal. Em geral, os modelos justificam pouco da variação observada, principalmente das características de comprimento do rádio, da canela, do fêmur, da tíbia, da quartela pélvica, e dos ângulos escapuloumeral, umerorradial, dedo torácico com a horizontal, tibiotarsometatarso e metatarsofalange, todos com coeficiente de determinação inferior a 0,20. Possivelmente, as dificuldades práticas para mensuração dos animais contribuiu para a maior variação das características angulares. 


\section{Lage et al.}

Tabela 2. Médias ajustadas pelo método dos quadrados mínimosterros-padrão e níveis de significância (P) das das medidas lineares e angulares de equinos da raça Mangalarga Marchador

\begin{tabular}{|c|c|c|c|c|c|c|c|c|c|c|}
\hline \multirow{2}{*}{$\begin{array}{l}\text { Característica } \\
\text { Linear }(\mathrm{cm})\end{array}$} & \multirow{2}{*}{$\mathrm{R}^{2}$} & \multicolumn{3}{|c|}{ Estado } & \multicolumn{3}{|c|}{ Tipo de criação } & \multicolumn{3}{|c|}{ Sexo } \\
\hline & & MG & SP & $\mathrm{P}$ & Baia & Pasto & $\mathrm{P}$ & Macho & Fêmea & $\mathrm{P}$ \\
\hline $\begin{array}{l}\text { Comprimento } \\
\text { da escápula }\end{array}$ & 0,35 & $60,0 \pm 0,002$ & $60,0 \pm 0,003$ & 0,98 & $61,0 \pm 0,006$ & $60,0 \pm 0,006$ & 0,73 & $59,0 \pm 0,005$ & $62,0 \pm 0,007$ & 0,06 \\
\hline $\begin{array}{l}\text { Comprimento } \\
\text { do úmero }\end{array}$ & 0,34 & $34,0 \pm 0,001$ & $33,0 \pm 0,002$ & $<0,001$ & $34,0 \pm 0,004$ & $34,0 \pm 0,004$ & 0,69 & $33,0 \pm 0,003$ & $35,0 \pm 0,004$ & 0,03 \\
\hline $\begin{array}{l}\text { Comprimento } \\
\text { do rádio }\end{array}$ & 0,09 & $35,0 \pm 0,002$ & $35,0 \pm 0,003$ & 0,87 & $35,0 \pm 0,005$ & $35,0 \pm 0,005$ & 0,81 & $34,0 \pm 0,004$ & $35,0 \pm 0,005$ & 0,63 \\
\hline $\begin{array}{l}\text { Comprimento } \\
\text { da canela }\end{array}$ & 0,16 & $9,0 \pm 0,0008$ & $9,0 \pm 0,001$ & 0,14 & $9,0 \pm 0,002$ & $9,0 \pm 0,002$ & 0,47 & $8,0 \pm 0,002$ & $9,0 \pm 0,002$ & 0,10 \\
\hline $\begin{array}{l}\text { Comprimento } \\
\text { do metacarpo }\end{array}$ & 0,28 & $22,0 \pm 0,002$ & $22,0 \pm 0,002$ & 0,64 & $21,0 \pm 0,004$ & $22,0 \pm 0,004$ & 0,68 & $21,0 \pm 0,004$ & $22,0 \pm 0,005$ & 0,76 \\
\hline $\begin{array}{l}\text { Comprimento } \\
\text { da quartela } \\
\text { torácica }\end{array}$ & 0,23 & $11,4 \pm 0,0008$ & $10,9 \pm 0,001$ & $<0,001$ & $11,5 \pm 0,002$ & $10,7 \pm 0,002$ & 0,04 & $11,6 \pm 0,002$ & $10,7 \pm 0,002$ & 0,03 \\
\hline $\begin{array}{l}\text { Comprimento } \\
\text { da garupa ou } \\
\text { coxal }\end{array}$ & 0,26 & $54,0 \pm 0,002$ & $54,0 \pm 0,003$ & 0,55 & $55,0 \pm 0,005$ & $53,0 \pm 0,005$ & 0,30 & $54,0 \pm 0,005$ & $54,0 \pm 0,006$ & 0,74 \\
\hline $\begin{array}{l}\text { Comprimento } \\
\text { do fêmur }\end{array}$ & 0,15 & $36,0 \pm 0,002$ & $35,0 \pm 0,003$ & 0,17 & $37,0 \pm 0,006$ & $34,0 \pm 0,0007$ & 0,01 & $37,0 \pm 0,006$ & $34,0 \pm 0,007$ & 0,06 \\
\hline $\begin{array}{l}\text { Comprimento } \\
\text { da tíbia }\end{array}$ & 0,18 & $31,0 \pm 0,005$ & $30,0 \pm 0,007$ & 0,21 & $29,0 \pm 0,01$ & $32,0 \pm 0,01$ & 0,33 & $29,0 \pm 0,01$ & $32,0 \pm 0,01$ & 0,33 \\
\hline $\begin{array}{l}\text { Comprimento } \\
\text { do tarso }\end{array}$ & 0,48 & $10,0 \pm 0,001$ & $11,0 \pm 0,001$ & $<0,001$ & $11,0 \pm 0,003$ & $9,0 \pm 0,003$ & 0,01 & $11,0 \pm 0,002$ & $9,0 \pm 0,003$ & 0,01 \\
\hline $\begin{array}{l}\text { Comprimento } \\
\text { do metatarso }\end{array}$ & 0,24 & $28,0 \pm 0,002$ & $27,0 \pm 0,003$ & 0,04 & $27,0 \pm 0,005$ & $28,0 \pm 0,005$ & 0,55 & $27,0 \pm 0,005$ & $28,0 \pm 0,006$ & 0,23 \\
\hline $\begin{array}{l}\text { Comprimento } \\
\text { da quartela } \\
\text { pélvica }\end{array}$ & 0,16 & $12,0 \pm 0,001$ & $11,0 \pm 0,002$ & $<0,01$ & $11,0 \pm 0,003$ & $11,0 \pm 0,003$ & 0,63 & $11,0 \pm 0,003$ & $11,0 \pm 0,003$ & 0,56 \\
\hline $\begin{array}{l}\text { Altura na } \\
\text { cernelha }\end{array}$ & 0,30 & $150,0 \pm 0,003$ & $150,0 \pm 0,004$ & 0,12 & $150,0 \pm 0,008$ & $150,0 \pm 0,008$ & 0,66 & $149,0 \pm 0,007$ & $151,0 \pm 0,009$ & 0,12 \\
\hline $\begin{array}{l}\text { Altura na } \\
\text { garupa }\end{array}$ & 0,20 & $148,0 \pm 0,003$ & $149,0 \pm 0,004$ & 0,08 & $149,0 \pm 0,008$ & $147,0 \pm 0,008$ & 0,26 & $148,0 \pm 0,007$ & $148,0 \pm 0,009$ & 0,98 \\
\hline $\begin{array}{l}\text { Perímetro da } \\
\text { canela }\end{array}$ & 0,32 & $18,0 \pm 0,0008$ & $18,0 \pm 0,001$ & 0,403 & $18,0 \pm 0,002$ & $18,0 \pm 0,002$ & 0,25 & $17,0 \pm 0,002$ & $18,0 \pm 0,002$ & 0,24 \\
\hline $\begin{array}{l}\text { Perímetro } \\
\text { torácico }\end{array}$ & 0,27 & $176,0 \pm 0,007$ & $180,0 \pm 0,009$ & $<0,001$ & $182,0 \pm 0,02$ & $175,0 \pm 0,02$ & 0,06 & $181,0 \pm 0,02$ & $176,0 \pm 0,02$ & 0,15 \\
\hline \multicolumn{11}{|l|}{$\begin{array}{l}\begin{array}{l}\text { Angular } \\
\text { (graus) }\end{array} \\
\end{array}$} \\
\hline $\begin{array}{l}\text { Escápula com } \\
\text { horizontal } \\
\text { Escapuloumera }\end{array}$ & 0,19 & $61,36 \pm 0,35$ & $62,46 \pm 0,46$ & $<0,01$ & $63,11 \pm 0,83$ & $60,71 \pm 0,87$ & 0,13 & $63,85 \pm 0,80$ & $59,97 \pm 0,97$ & 0,02 \\
\hline 1 & 0,32 & $88,90 \pm 0,70$ & $84,85 \pm 0,92$ & $<0,001$ & $86,61 \pm 1,67$ & $87,13 \pm 1,75$ & 0,87 & $87,15 \pm 1,62$ & $86,60 \pm 1,96$ & 0,87 \\
\hline Úmerorradial & 0,12 & $130,53 \pm 0,67$ & $129,71 \pm 0,89$ & 0,42 & $128,62 \pm 1,62$ & $131,62 \pm 1,70$ & 0,33 & $129,30 \pm 1,56$ & $130,95 \pm 1,90$ & 0,61 \\
\hline $\begin{array}{l}\text { Metacarpo- } \\
\text { falange }\end{array}$ & 0,28 & $147,89 \pm 0,74$ & $144,76 \pm 0,98$ & $<0,01$ & $146,15 \pm 1,77$ & $146,49 \pm 1,85$ & 0,92 & $143,76 \pm 1,72$ & $148,89 \pm 2,09$ & 0,15 \\
\hline $\begin{array}{l}\text { Dedo torácico } \\
\text { c/ a horizontal }\end{array}$ & 0,19 & $55,50 \pm 0,62$ & $55,07 \pm 0,82$ & 0,64 & $56,60 \pm 1,48$ & $54,97 \pm 1,55$ & 0,35 & $55,22 \pm 1,43$ & $55,35 \pm 1,74$ & 0,97 \\
\hline Coxal & 0,44 & $54,34 \pm 0,47$ & $50,30 \pm 0,62$ & $<0,001$ & $52,45 \pm 1,13$ & $52,19 \pm 1,18$ & 0,91 & $53,26 \pm 1,09$ & $51,38 \pm 1,33$ & 0,41 \\
\hline Coxofemoral & 0,32 & $138,37 \pm 0,73$ & $134,13 \pm 0,97$ & $<0,001$ & $137,37 \pm 1,75$ & $135,12 \pm 1,83$ & 0,50 & $138,66 \pm 1,69$ & $133,83 \pm 2,06$ & 0,17 \\
\hline Femoro-tibial & 0,23 & $149,92 \pm 0,59$ & $151,40 \pm 0,78$ & 0,09 & $152,75 \pm 1,41$ & $148,57 \pm 1,48$ & 0,12 & $153,57 \pm 1,37$ & $147,76 \pm 1,66$ & 0,04 \\
\hline $\begin{array}{l}\text { Tibiotarsico- } \\
\text { metatársico }\end{array}$ & 0,15 & $152,58 \pm 0,68$ & $155,65 \pm 0,91$ & $<0,01$ & $157,16 \pm 1,64$ & $151,08 \pm 1,71$ & 0,05 & $158,06 \pm 1,58$ & $150,17 \pm 1,93$ & 0,02 \\
\hline $\begin{array}{l}\text { Metatarso- } \\
\text { falange }\end{array}$ & 0,11 & $153,49 \pm 1,24$ & $152,64 \pm 1,64$ & 0,64 & $156,17 \pm 2,96$ & $149,95 \pm 3,10$ & 0,27 & $156,78 \pm 2,86$ & $149,34 \pm 3,48$ & 0,21 \\
\hline $\begin{array}{l}\text { Dedo pélvico } \\
\text { c/ a horizontal }\end{array}$ & 0,25 & $60,11 \pm 0,65$ & $57,61 \pm 0,86$ & 0,01 & $58,28 \pm 1,55$ & $59,44 \pm 1,62$ & 0,70 & $56,24 \pm 1,50$ & $61,48 \pm 1,83$ & 0,10 \\
\hline
\end{tabular}

As medidas lineares que apresentaram efeito significativo do estado de origem do criatório foram as do comprimento do úmero, das quartelas torácica e pélvica, do tarso e do metatarso e a do perímetro torácico, que representaram $37,5 \%$ das 16 características avaliadas. Dentre as 11 medidas angulares, quatro $(36,4 \%)$ não evidenciaram tal efeito, os 
ângulos umerorradial, dedo torácico com a horizontal, femorotibial e metatarsofalangeano. A diferença entre os animais dos estados de Minas Gerais e São Paulo, tanto para as características lineares quanto para as angulares, não manteve consistência de maiores ou menores mensurações de um estado em relação ao outro. Em razão disso, torna-se difícil conjecturar sobre as diferenças observadas para o conjunto das características. Entretanto, algumas comparações entre características específicas podem esclarecer certas particularidades dos criatórios desses dois estados. Observaram-se maiores valores das medidas de comprimento do úmero, das quartelas torácica e pélvica e do metatarso; dos ângulos escapuloumeral, metacarpofalange, coxal, coxofemoral e dedo pélvico com a horizontal nos animais do Estado de Minas Gerais. Os do Estado de São Paulo apresentaram maiores valores para comprimento do tarso, perímetro torácico, ângulo escápulo-horizontal e ângulo tibiotarsometatarso. Em geral, nos animais do Estado de Minas Gerais, as características lineares e angulares dos membros torácicos foram maiores que nos de São Paulo. Para ângulos dos membros pélvicos, os de Minas Gerais apresentaram maiores valores para três medidas e os de São Paulo, para uma característica. O maior valor do comprimento dos ossos dos membros torácicos dos animais de Minas Gerais não resultou em maior altura na cernelha. Isto pode ser atribuído à relação entre os valores dos ângulos e dos comprimentos dos ossos dos membros torácicos, o que resultou em valores de altura na cernelha próximos entre os animais dos dois estados. Também, pode-se aventar como explicação para essas variações as possíveis diferenças regionais na escolha dos atributos morfométricos. $\mathrm{O}$ maior perímetro torácico dos animais de São Paulo indica maior abertura torácica, condição que tem como fatores adjuvantes a genética, a nutrição e o condicionamento.

O efeito de criatório dentro de Estado foi observado para as medidas lineares de comprimento do úmero, do metacarpo, do coxal, da tíbia, do tarso e do metatarso e a medida do perímetro torácico. Os ângulos influenciados por esse efeito foram os escapuloumeral, metacarpofalangeano, coxal, coxofemoral e femorotibial. Esses resultados podem também ser explicados pelas hipóteses anteriormente levantadas, como diferenças entre criadores quanto ao direcionamento na escolha de reprodutores e diversidade de ambiente como a nutrição e os programas de treinamento.

O tipo de criação (pasto ou baia) apresentou efeito significativo apenas para três medidas lineares $(11,0 \%)$, os comprimentos da quartela torácica, do fêmur e do tarso. Deve-se considerar que, no presente estudo, os sistemas de criação, no que se relaciona aos referenciais de nutrição e, principalmente, ao tempo de permanência em baia ou pasto, não foram determinados com exatidão. Tal fato dificulta comentários e conclusões a partir dos resultados obtidos. Observou-se efeito significativo de sexo sobre os comprimentos do úmero, da quartela torácica e do tarso e sobre os ângulos da escápula com a horizontal, do femorotibial e do tibiotarsometetarso, isto é, 22,2\% das 27 medidas analisadas. As medidas de comprimento da escápula, do úmero, do rádio, da canela, da tíbia e do metacarpo e as do perímetro torácico foram maiores nas fêmeas, fato não observado para as características angulares. Esses resultados podem ser consequência do regime nutricional e de treinamento diferenciado impostos aos animais dos dois sexos. Nos criatórios estudados, os machos, criados presos, em geral estão mais sujeitos a dietas inadequadas. A ausência ou a limitação de exercícios físicos, principalmente na fase de crescimento, aliado a esses problemas de nutrição, necessários para o completo desenvolvimento do animal, predispõem a permanentes alterações ortopédicas e de desenvolvimento corporal dos animais. Arnason (1984) também observou que o sexo influenciou todas as medidas corporais de animais da raça Icelandic Toelter na Islândia.

$\mathrm{O}$ efeito de idade foi significativo sobre quatro $(14,8 \%)$ medidas: as de comprimento do metacarpo, do coxal e do metatarso, e a do perímetro da canela, cujas equações de regressão são apresentadas na Tab. 3 . 
Tabela 3. Coeficientes de regressão \pm erro-padrão $(b \pm E P)$ e níveis de significância $(\mathrm{P})$ das medidas lineares e angulares sobre a idade (mes) de equinos da raça Mangalarga Marchador

\begin{tabular}{|c|c|c|c|c|}
\hline \multirow{2}{*}{$\begin{array}{l}\text { Característica } \\
\text { Linear }(\mathrm{cm})\end{array}$} & \multicolumn{2}{|c|}{ Coeficientes } & \multirow{2}{*}{ EP } & \multirow{2}{*}{$\mathrm{P}$} \\
\hline & Intercepto & $\mathrm{b}$ & & \\
\hline Comprimento da escápula & 0,6236 & $-0,0032$ & 0,0033 & 0,37 \\
\hline Comprimento do úmero & 0,3486 & $-0,0012$ & 0,0025 & 0,60 \\
\hline Comprimento do rádio & 0,3526 & 0,0004 & 0,0015 & 0,90 \\
\hline Comprimento da canela & 0,0956 & 0,0013 & 0,0015 & 0,29 \\
\hline Comprimento do metacarpo & 0,2031 & $-0,0102$ & 0,0412 & 0,04 \\
\hline $\begin{array}{l}\text { Comprimento da quartela } \\
\text { torácica }\end{array}$ & 0,1015 & $-0,0010$ & 0,0024 & 0,80 \\
\hline Comprimento da garupa ou coxal & 0,5460 & $-0,0067$ & 0,0018 & 0,03 \\
\hline Comprimento do fêmur & 0,3129 & 0,0063 & 0,0054 & 0,21 \\
\hline Comprimento da tíbia & 0,3214 & 0,0052 & 0,0078 & 0,99 \\
\hline Comprimento do tarso & 0,0914 & 0,0051 & 0,0042 & 0,06 \\
\hline Comprimento do metatarso & 0,2861 & $-0,0071$ & 0,0052 & 0,03 \\
\hline Comprimento da quartela pélvica & 0,1135 & 0,0012 & 0,0014 & 0,52 \\
\hline Altura na cernelha & 1,53 & $-0,0066$ & 0,0051 & 0,19 \\
\hline Altura na garupa & 1,49 & $-0,0068$ & 0,0049 & 0,17 \\
\hline Perímetro da canela & 0,17 & 0,0034 & 0,0012 & $<0,01$ \\
\hline Perímetro torácico & 1,77 & $-0,0027$ & 0,0114 & 0,81 \\
\hline \multicolumn{5}{|l|}{ Angular (graus) } \\
\hline Escápula com horizontal & 60,67 & $-0,0041$ & 0,0053 & 0,44 \\
\hline Escapuloumeral & 84,50 & 0,0053 & 0,0107 & 0,62 \\
\hline Umerorradial & 130,76 & 0,0075 & 0,0103 & 0,47 \\
\hline Metacarpofalange & 147,92 & 0,0031 & 0,0113 & 0,78 \\
\hline Dedo torácico com a horizontal & 56,25 & $-0,0114$ & 0,0094 & 0,22 \\
\hline Coxal & 48,65 & 0,0029 & 0,0072 & 0,68 \\
\hline Coxofemoral & 130,55 & $-0,0115$ & 0,0112 & 0,30 \\
\hline Femorotibial & 147,78 & $-0,0085$ & 0,0090 & 0,34 \\
\hline Tibiotarsicometatársico & 150,41 & $-0,0091$ & 0,0105 & 0,38 \\
\hline Metatarsofalange & 145,91 & $-0,0216$ & 0,0189 & 0,25 \\
\hline Dedo pélvico com a horizontal & 62,79 & $-0,0066$ & 0,0099 & 0,50 \\
\hline
\end{tabular}

Observou-se redução de, aproximadamente, $0,2 \%$ do comprimento do metacarpo associada ao aumento de um mês de idade do animal. Para o comprimento coxal (garupa) e o do metatarso, essa relação foi aproximadamente de $0,9 \%$ e $2,6 \%$, respectivamente, a cada mês de idade do animal. A aparente pouca importância do efeito da idade sobre as características estudadas pode estar associada à maturidade óssea dos animais estudados, pois o grupo foi composto por animais adultos, com média de idade de 92 meses. O fechamento da porção distal da cartilagem epifisária do metacarpo e do metatarso ocorre entre nove e 18 meses. Para o ílio, ísquio e púbis, o período de fusão ocorre entre 10 e 12 meses; para os centros secundários da crista, do tubérculo coxal, do isquiático e da porção acetabular do púbis, as placas de crescimento se fecham entre quatro e cinco anos de idade. Zamborlini et al. (1996), ao estimarem os efeitos do ambiente e dos parâmetros genéticos das medidas corporais e da pontuação obtida à época do registro definitivo de animais Mangalarga Marchador, observaram efeito significativo do sexo, ano e idade dos animais, em todas as medidas corporais, exceto para o comprimento da cabeça. No trabalho desses autores, a média de idade era inferior à do atual estudo.

$\mathrm{Na}$ Tab. 4, são apresentadas as associações obtidas entre o comprimento ósseo e as medidas angulares. 
Tabela 4. Coeficientes de correlação de resíduos entre o comprimento dos ossos e os ângulos dos membros torácicos e pélvicos de equinos da raça Mangalarga Marchador

\begin{tabular}{cccccccccccc}
\hline Característica & AEH & AEU & AUR & AMCF & ADH & ACX & ACF & AFT & ATTM & AMTF & ADHP \\
\hline CES & $-0,07$ & $-0,02$ & 0,02 & 0,01 & 0,02 & 0,03 & $-0,07$ & $-0,04$ & 0,007 & 0,08 & $0,18^{*}$ \\
CUM & $-0,10$ & $-0,09$ & $-0,05$ & $-0,04$ & 0,01 & $-0,15^{*}$ & $-0,05$ & 0,05 & 0,02 & 0,04 & $-0,12$ \\
CRA & $-0,04$ & 0,01 & $-0,18^{*}$ & $-0,06$ & 0,11 & $-0,05$ & 0,05 & 0,14 & 0,007 & 0,07 & 0,09 \\
CCA & 0,12 & 0,02 & $-0,02$ & $-0,09$ & $-0,21^{* *}$ & 0,03 & $-0,07$ & $-0,06$ & 0,08 & $0,16^{*}$ & $-0,02$ \\
CMC & $-0,22^{* *}$ & $-0,13$ & $-0,11$ & $-0,09$ & 0,03 & $-0,03$ & 0,01 & 0,002 & $-0,02$ & $0,16^{*}$ & $-0,03$ \\
CQU & $-0,03$ & 0,04 & $-0,007$ & $-0,07$ & $-0,06$ & $-0,09$ & $-0,10$ & 0,03 & $-0,04$ & 0,10 & 0,11 \\
CCX & $-0,009$ & 0,03 & 0,08 & 0,11 & 0,09 & $-0,12$ & 0,05 & $-0,01$ & 0,03 & 0,13 & $0,17^{*}$ \\
CFE & $-0,01$ & $-0,06$ & $0,17^{*}$ & $-0,08$ & $-0,01$ & $-0,33^{* *}$ & $-0,16^{*}$ & 0,08 & $0,17^{*}$ & 0,02 & $-0,07$ \\
CTI & 0,10 & 0,05 & $-0,003$ & $-0,11$ & 0,03 & 0,0006 & $-0,06$ & $-0,15$ & $-0,06$ & 0,02 & 0,05 \\
CTA & 0,07 & 0,04 & 0,04 & $-0,16^{*}$ & $-0,18^{*}$ & $-0,12$ & 0,01 & 0,09 & 0,008 & $-0,05$ & $-0,06$ \\
CMT & $-0,12$ & 0,02 & $-0,04$ & $-0,05$ & 0,01 & 0,11 & 0,08 & 0,07 & 0,02 & $-0,05$ & $-0,08$ \\
CQP & $-0,01$ & $-0,01$ & $-0,11$ & $-0,01$ & $-0,11$ & $-0,05$ & 0,03 & 0,09 & 0,03 & $0,17^{*}$ & $0,17^{*}$ \\
\hline
\end{tabular}

P $<0,05 ;{ }^{* *} \mathrm{P}<0,005$.

CES: comprimento da escápula; CUM: comprimento do úmero; CRA: comprimento do rádio; CCA: comprimento da canela; CMC: comprimento do metacarpo; CQU: comprimento da quartela torácica; CCX: comprimento coxal (garupa); CFE: comprimento do fêmur; CTI: comprimento da tíbia; CTA: comprimento do tarso; CMT: comprimento do metatarso; CQP: comprimento da quartela pélvica; AEH: ângulo escápula com a horizontal; AEU: ângulo escapuloumeral; AUR: ângulo umerorradial; AMCF: ângulo metacarpofalange, $\mathrm{ADH}$ : ângulo dedo torácico com a horizontal; ACX: ângulo coxal; ACF: ângulo coxofemoral; AFT: ângulo femorotibial; ATTM: ângulo tibiotarsometatarso; AMTF: ângulo metatarsofalange, ADHP: ângulo dedo pélvico com a horizontal.

Apenas $15(11,4 \%)$ das 132 associações possíveis entre o comprimento dos ossos e as medidas angulares apresentaram correlação diferente de zero, com variação de $-0,33$ a 0,18 . Destas, sete $(46,7 \%)$ apresentaram valores entre 0,16 (comprimento da canela anterior e ângulo metatarsofalangeano) e 0,18 (comprimento da escápula e ângulo do dedo posterior com a horizontal) e as oito associações restantes apresentaram valores negativos de correlação, de 0,33 (comprimento do fêmur e ângulo coxal) a 0,15 (comprimento do úmero e ângulo coxal). Holmström et al. (1990) obtiveram correlações fenotípicas positivas entre o comprimento das falanges dos membros pélvicos e o ângulo tibiotarsicometatatarso, evidenciando, também, associação com a funcionalidade dos membros pélvicos. No presente estudo, observou-se que o comprimento do fêmur foi a característica que apresentou associação diferente de zero com o maior número de medidas angulares. $\mathrm{O}$ aumento do comprimento do fêmur estava relacionado a maiores ângulos umerorradial e tibiotarsometatarso, e o incremento desse osso implicou menores medidas para os ângulos coxal e coxofemoral. Confirmando a relação entre o comprimento e a direção da quartela pélvica, observou-se que, à medida que houve aumento do comprimento do fêmur, houve incremento dos ângulos metatarsofalange e dedo posterior com a horizontal. A avaliação das associações, de forma geral, mostrou que essas estão relacionadas à morfofisiologia dos ossos e suas articulações, com suas respectivas direções. Esse posicionamento confere aos ângulos dos membros torácicos e pélvicos funções de sustentação, impulsão e permitem os movimentos de flexão e extensão.

As associações entre as 11 medidas angulares obtidas no estudo estão apresentadas na Tab. 5.

Dentre as 55 associações possíveis entre as medidas angulares, $11(20 \%)$ apresentaram correlação diferente de zero, sendo 10 positivas, com valores entre 0,15 (ângulos coxal e metatarsofalangeano) e 0,40 (ângulos metacarpofalangeano e dedo torácico com a horizontal, ângulos coxal e coxofemural). Correlação com sinal negativo foi observada entre os ângulos escapuloumeral e femorotibial, -0,18. Estas associações confirmam as relações existentes entre os ângulos corporais conforme a morfofisiologia do cavalo, em que a direção dos ossos dos membros torácicos e pélvicos está relacionada à função de sustentação, amortecimento, impulsão e propulsão (Back e Clayton, 2001). Além disso, para a harmonia morfológica do animal e a fim de que os andamentos sejam regulares, é importante a relação 
entre as aberturas dos ângulos dos membros torácicos e pélvicos, respeitando os valores conforme a aptidão do animal. Para tal, a variação entre os ângulos dos membros deve guardar certa correlação (Camargo e Chieffi, 1971; Nascimento, 1999; Back e Clayton, 2001). Nota-se que a abertura dos ângulos metacarpofalange, dedos torácicos e pélvicos e tibiotarsometatarso mantêm relação positiva, em que o incremento de um desses está associado ao concomitante aumento dos outros. Essas associações foram também obtidas entre os ângulos coxal, coxo-femoral e metatarsofalange; femorotibial e tibiotarsometatarso e entre os ângulos tibiotarsometatarso e dedo pélvico com a horizontal.

Na Tab. 6, são apresentadas as associações obtidas entre os comprimentos dos ossos.

Tabela 5. Coeficientes de correlação de resíduos entre ângulos dos membros torácicos e pélvicos de equinos da raça Mangalarga Marchador

\begin{tabular}{ccccccccccc}
\hline Característica & AEU & AUR & AMCF & ADH & ACX & ACF & AFT & ATTM & AMTF & ADHP \\
\hline AEH & 0,008 & $0,16^{*}$ & $-0,06$ & $-0,08$ & $-0,02$ & $-0,07$ & 0,07 & $-0,004$ & 0,01 & $-0,02$ \\
AEU & 1,00 & 0,14 & 0,06 & $-0,008$ & 0,08 & $-0,14$ & $-0,18^{*}$ & $-0,05$ & $-0,004$ & 0,02 \\
AUR & & 1,00 & $-0,12$ & $-0,05$ & 0,03 & 0,05 & $-0,09$ & $-0,07$ & $-0,05$ & 0,03 \\
AMCF & & & 1,00 & $0,40^{* *}$ & $-0,05$ & $-0,07$ & $-0,10$ & $0,26^{* *}$ & 0,06 & $0,20^{* *}$ \\
ADH & & & & 1,00 & $-0,02$ & $-0,05$ & $-0,03$ & $-0,05$ & 0,13 & $0,20^{*}$ \\
ACX & & & & & 1,00 & $0,40^{* *}$ & $-0,05$ & $-0,04$ & $0,15^{*}$ & 0,12 \\
ACF & & & & & & 1,00 & $0,23^{* *}$ & 0,11 & 0,10 & 0,02 \\
AFT & & & & & & & 1,00 & $0,35^{* *}$ & $-0,02$ & $-0,13$ \\
ATTM & & & & & & & & 1,00 & $-0,03$ & $-0,12$ \\
AMTF & & & & & & & & & 1,00 & $0,20^{*}$ \\
\hline
\end{tabular}

${ }^{*} \mathrm{P}<0,05 ;{ }^{* *} \mathrm{P}<0,005$.

AEH: ângulo escápula com a horizontal; AEU: ângulo escapuloumeral; AUR: ângulo umerorradial; AMCF: ângulo metacarpofalange; ADH: ângulo dedo torácico com a horizontal; ACX: ângulo coxal; ACF: ângulo coxofemoral; AFT: ângulo femorotibial; ATTM: ângulo tibiotarsometatarso; AMTF: ângulo metatarsofalange; ADHP: ângulo dedo pélvico com a horizontal.

Tabela 6. Coeficientes de correlação de resíduos entre medidas de comprimento dos ossos dos membros de equinos da raça Mangalarga Marchador

\begin{tabular}{cccccccccccc}
\hline Característica & CUM & CRA & CCA & CMC & CQU & CCX & CFE & CTI & CTA & CMT & CQP \\
\hline CES & $0,35^{* *}$ & 0,11 & 0,03 & 0,10 & $0,19^{*}$ & $0,46^{* *}$ & 0,12 & $0,23^{* *}$ & $-0,05$ & $0,26^{* *}$ & $0,21^{*}$ \\
CUM & 1,00 & $0,30^{* *}$ & 0,06 & $0,22^{* *}$ & $0,27^{* *}$ & $0,43^{* *}$ & $0,29^{*}$ & $0,18^{*}$ & 0,04 & $0,19^{*}$ & $0,16^{*}$ \\
CRA & & 1,00 & $-0,36^{* *}$ & 0,05 & 0,11 & $0,26^{* *}$ & $-0,14$ & 0,12 & $-0,12$ & $0,28^{* *}$ & $0,21^{*}$ \\
CCA & & & 1,00 & $-0,16^{*}$ & $-0,04$ & 0,02 & $0,22^{* *}$ & 0,02 & 0,12 & 0,001 & 0,07 \\
CMC & & & & 1,00 & $0,18^{*}$ & 0,05 & 0,01 & 0,03 & 0,13 & $-0,02$ & 0,002 \\
CQU & & & & & 1,00 & 0,11 & 0,10 & $-0,03$ & 0.007 & $-0,02$ & $0,17^{*}$ \\
CCX & & & & & & 1,00 & $0,19^{*}$ & 0,10 & $-0,004$ & $0,17^{*}$ & $0,16^{*}$ \\
CFE & & & & & & & 1,00 & 0,01 & $0,21^{* *}$ & 0,02 & 0,05 \\
CTI & & & & & & & & 1,00 & $-0,07$ & 0,10 & 0,02 \\
CTA & & & & & & & & & 1,00 & $0,26^{* *}$ & 0,02 \\
CMT & & & & & & & & & 1,00 & 0,13 \\
\hline
\end{tabular}

${ }^{*} \mathrm{P}<0,05 ;{ }^{* *} \mathrm{P}<0,005$.

CES: comprimento da escápula; CUM: comprimento do úmero; CRA: comprimento do rádio; CCA: comprimento da canela: CMC: comprimento do metacarpo; CQU: comprimento da quartela torácica; CCX: comprimento coxal (garupa); CFE: comprimento do fêmur; CTI: comprimento da tíbia; CTA: comprimento do tarso; CMT: comprimento do metatarso; CQP: comprimento da quartela pélvica. 
Vinte e seis $(39,4 \%)$ das 66 associações possíveis entre as medidas de comprimento dos ossos dos membros apresentaram valores diferentes de zero, sendo $24(92,3 \%)$ positivas, com variação de 0,16 (comprimentos do úmero e do coxal com a quartela pélvica) a 0,46 (comprimentos da escápula e coxal). Correlações negativas foram observadas apenas entre as medidas de comprimento da canela e do metatarso, $-0,16$, e entre o comprimento do rádio e da canela, -0,36. $\mathrm{Na}$ raça Andaluz, Molina et al. (1999) obtiveram correlações fenotípicas de 0,15 (perímetro dos ossos do carpo e altura na cernelha) a 0,60 (circunferência do tórax e altura na cernelha). A associação observada entre os comprimentos da escápula e da garupa $(0,46)$ confirmou os achados de Zamborlini (1996), indicando a relação entre essas características e o papel morfofisiológico realizado pela escápula para a transformação da força dos membros pélvicos em rendimento. Isso ressalta a importância da escápula em equinos como imprescindível à dinâmica: ela deve ser larga, musculosa e com movimentos amplos, que proporcionem andamento cômodo e regular (Nascimento, 1999). Deve-se considerar que o comprimento da escápula está intrinsecamente relacionado à conformação da cernelha, do tronco e dos membros, que, aliado à sua inclinação, constituem fatores de importância na biomecânica dos movimentos da cintura torácica. Isto é válido, também, para o eixo umeral. Quando proporcional, possibilita maior amplitude de movimentos, pela sua oscilação e maior área útil para inserção muscular, o que permite movimentos amplos e de maior flexibilidade, além de caixa torácica mais alta e com melhor proteção (Harris, 1993; Sierra et al., 1998; Nascimento, 1999).

O comprimento do úmero foi a medida que apresentou maior número de associações positivas com as outras medidas lineares, e está associado aos comprimentos do rádio, do metacarpo, das quartelas, do coxal, do fêmur e da tíbia. Pode-se afirmar que a escolha de animais que apresentem úmero mais comprido está associada à escolha de animais com ossos mais longos dos membros torácico e pélvico.

$\mathrm{Na}$ Tab. 7, são apresentadas as associações obtidas entre o comprimento dos ossos e as medidas de alturas e perímetros.

Tabela 7. Coeficientes de correlação de resíduos entre comprimento dos ossos e medidas de alturas e perímetros de equinos da raça Mangalarga Marchador

\begin{tabular}{ccccccccccccc}
\hline Característica & CES & CUM & CRA & CCA & CMC & CQU & CCX & CFE & CTI & CTA & CMT & CQP \\
\hline ALC & $0,57^{* *}$ & $0,51^{* *}$ & $0,41^{* *}$ & 0,07 & $0,20^{*}$ & $0,31^{* *}$ & $0,59^{* *}$ & $0,28^{*}$ & $0,19^{*}$ & 0,13 & $0,26^{* *}$ & $0,37^{* *}$ \\
ALG & $0,46^{* * *}$ & $0,49^{*}$ & $0,39^{* *}$ & 0,11 & $0,26^{* *}$ & $0,35^{* *}$ & $0,57^{*}$ & $0,33^{* * *}$ & 0,11 & 0,12 & $0,27^{* *}$ & $0,36^{* *}$ \\
PCA & $0,22^{* *}$ & $0,34^{* *}$ & 0,13 & 0,13 & 0,01 & $0,25^{* *}$ & $0,34^{* * *}$ & $0,23^{* *}$ & 0,13 & $-0,03$ & 0,13 & 0,10 \\
PTO & $0,46^{* *}$ & 0,34 & 0,10 & 0,10 & 0,08 & 0,06 & 0,65 & $0,19^{* *}$ & 0,14 & $-0,03$ & $0,20^{*}$ & 0,14 \\
\hline
\end{tabular}

"P<0,05; " $\mathrm{P}<0,005$.

ALC: altura na cernelha; ALG: altura na garupa; PCA: perímetro da canela; PTO: perímetro torácico; CES: comprimento da escápula; CUM: comprimento do úmero; CRA: comprimento do rádio; CCA: comprimento da canela; CMC: comprimento do metacarpo; CQU: comprimento da quartela torácica; CCX: comprimento coxal (garupa); CFE: comprimento do fêmur; CTI: comprimento da tíbia; CTA: comprimento do tarso; CMT: comprimento do metatarso; CQP: comprimento da quartela pélvica.

Das 48 associações testadas entre o comprimento dos ossos e as medidas de alturas e perímetros, 28 $(58,3 \%)$ apresentaram correlações importantes e positivas, com variação de 0,19 (altura na cernelha com comprimento da tíbia) a 0,59 (altura na cernelha e comprimento coxal). Tal resultado era esperado, pois essas medidas retratam o sincronismo do crescimento do animal e a relação entre comprimento dos ossos e as mensurações compostas (alturas e perímetros).
A altura na cernelha e na garupa apresentou associação positiva com os ossos dos membros torácico e pélvico, confirmando os resultados de Barbosa (1993) e Zamborlini (1996). O último autor obteve alto valor de correlação fenotípica entre alturas na cernelha e na garupa $(0,73)$, altura na cernelha e comprimento do corpo $(0,68)$ e associação com valor médio entre perímetro torácico e altura na cernelha $(0,44)$, enquanto Barbosa (1993) obteve os seguintes valores de correlação, para as respectivas medidas: 0,89 entre as alturas da cernelha e da garupa, 0,62 entre altura 
na cernelha e comprimento do corpo e 0,23 entre perímetro torácico e altura na cernelha. Segundo Holmström et al. (1990) e Saastamoinen (1990), são elevadas as correlações fenotípicas entre a altura na cernelha e demais medidas de comprimento do corpo dos equinos. Estas observações indicam que a escolha de equinos a partir da altura da cernelha e da garupa acarreta concomitante incremento do tamanho dos ossos dos membros e dos perímetros da canela e torácico, respeitando as associações e seus respectivos valores.

As associações obtidas entre os ângulos dos membros e medidas de alturas e perímetros são apresentadas na Tab. 8.

Apenas duas (4,5\%) dentre as 44 associações entre as medidas angulares e medidas de altura e perímetro apresentaram valores diferentes de zero. Entre altura na garupa e ângulo metatarsofalangeano, e entre altura na cernelha e ângulo dedo pélvico com a horizontal, as correlações foram, respectivamente, 0,16 e 0,20. Essas associações indicam que tanto a altura na cernelha quanto na garupa, bastante associadas entre si $(0,86)$, estão relacionadas a animais com maiores ângulos metatarsofalange e dedo pélvico com a horizontal.

Na Tab. 9, são apresentadas as associações obtidas entre as medidas de altura e perímetro. As associações entre essas medidas foram importantes e variaram de 0,35 , entre perímetro da canela e perímetro torácico, a 0,86 , entre as alturas de cernelha e garupa. Esses valores sinalizam que a escolha fenotípica de cavalos Mangalarga Marchador pela altura na cernelha implicará animais mais altos na garupa e com maiores perímetros torácico e da canela, constatações também obtidas por Barbosa (1993) e Zamborlini (1996).

Tabela 8. Coeficientes de correlação de resíduos entre ângulos dos membros e medidas de alturas e perímetros de equinos da raça Mangalarga Marchador

\begin{tabular}{cccccccccccc} 
Característica & AEH & AEU & AUR & AMCF & ADH & ACX & ACF & AFT & ATTM & AMTF & ADHP \\
\hline ALC & 0,05 & $-0,005$ & $-0,007$ & 0,03 & 0,13 & $-0,10$ & $-0,02$ & $-0,01$ & $-0,001$ & 0,13 & $0,20^{*}$ \\
ALG & 0,12 & $-0,05$ & $-0,002$ & 0,07 & 0,12 & $-0,05$ & $-0,002$ & 0,07 & 0,10 & $0,16^{*}$ & 0,14 \\
PCA & 0,04 & 0,04 & 0,05 & $-0,04$ & 0,14 & $-0,11$ & $-0,08$ & $-0,05$ & 0,02 & 0,06 & 0,06 \\
PTO & 0,10 & 0,04 & 0,05 & 0,08 & 0,05 & $-0,07$ & 0,01 & 0,009 & 0,15 & 0,12 & 0,08
\end{tabular}

${ }^{*} \mathrm{P}<0,05 ;{ }^{* *} \mathrm{P}<0,005$.

ALC: altura na cernelha; ALG: altura na garupa; PCA: perímetro da canela; PTO: perímetro torácico; AEH: ângulo escápula com a horizontal; AEU: ângulo escapuloumeral: AUR: ângulo úmero-radial; AMCF: ângulo metacarpofalange; ADH: ângulo dedo com a horizontal; ACX: ângulo coxal; ACF: ângulo coxofemoral; AFT: ângulo femorotibial; ATTM: ângulo tibiotarsometatarso; AMTF: ângulo metatarsofalange; ADHP: ângulo dedo pélvico com a horizontal.

Tabela 9. Coeficientes de correlação de resíduos entre as medidas de altura e perímetro de equinos da raça Mangalarga Marchador

\begin{tabular}{cccc}
\hline Características & ALG & PCA & PTO \\
\hline ALC & $0,86^{* *}$ & $0,41^{* *}$ & $0,45^{* *}$ \\
ALG & 1,00 & $0,48^{* *}$ & $0,43^{* *}$ \\
PCA & & 1,00 & $0,35^{* *}$ \\
\hline
\end{tabular}

${ }^{*} \mathrm{P}<0,05 ;{ }^{* *} \mathrm{P}<0,005$.

ALC: altura na cernelha; ALG: altura na garupa; PCA: perímetro da canela; PTO: perímetro torácico.

\section{CONCLUSÕES}

As fontes de variação associadas ao ambiente, idade à avaliação e sexo influenciaram significativamente diversas características, e os efeitos dessas fontes devem ser considerados para a escolha dos indivíduos quando baseada nas medidas lineares e angulares. A escolha de animais com úmero de maior comprimento está associada a ossos dos membros torácicos e pélvicos mais longos. A escolha de animais com base no do tamanho dos ossos dos membros acarretará concomitante incremento das alturas de cernelha, garupa e dos perímetros da canela e torácico. A escolha fenotípica dos animais considerando as medidas dos ângulos metacarpofalangeano e dedo torácico com a horizontal refletirá positivamente na altura da cernelha e da garupa.

\section{AGRADECIMENTOS}

Os autores agradecem à Associação Brasileira dos Criadores do Cavalo Margalarga Marchador por ter possibilitado o experimento, e ao CNPq e à FAPEMIG, pelo apoio financeiro. 


\section{REFERÊNCIAS BIBLIOGRÁFICAS}

COSTA, M.D.; BERGMANN, J.A.G.; PEREIRA, C.S. et al. Caracterização das proporções morfométricas dos pôneis da raça Brasileira. Arq. Bras. Med. Vet. Zootec., v.50, p.455-460, 1998.

COSTA, M.D.; BERGMANN, J.A.G.; REZENDE, A.S.C. Caracterização demográfica da raça Mangalarga Marchador. Arq. Bras. Med. Vet. Zootec., v.56, p.687-690, 2004.

ARNASON, T. Genetic studies on conformation and performance of Icelandic Toelter horses. 1 Estimation of non-genetic effects and genetic parameters. Acta Agric. Scand., v.34, p.409-427, 1984.

BACK, W.; CLAYTON, H. Equine Locomotion. London: W. B. Saunders, 2001. 384p.

BARBOSA, C.G. Estudo morfométrico na raça Mangalarga Marchador: uma abordagem multivariada. 1993. 77f. Dissertação (Mestrado) - Escola de Veterinária, Universidade Federal de Minas Gerais, Belo Horizonte.

CAMARGO, M.X.; CHIEFFI, A. Ezoognósia. São Paulo: Instituto de Zootecnia, 1971. 320p.

CARNEIRO, G.G.; FONTES, C.R.; PEREIRA, H.C. Informações preliminares sobre o cavalo Mangalarga Marchador. Arq. Esc. Sup. Vet. UREMG, v.5, p.5-9, 1952.

ESTATUTO da ABCCMM e regulamento do serviço de registro genealógico. Belo Horizonte: ABCCMM, 2001. 68p.

HARRIS, S.E. Horse gaits, balance and movement. New York: Wiley, 1993. 178p.

HICKMAN, J.; COLLLES, C. Measurement of horses. Vet. Rec., v.114, p.491-493, 1984.

HOLMSTRÖM, M.; MAGNUSON, L.E.; PHILIPSON, J. Variation in conformation of Swedish Warmblood horses and conformational characteristics of elite sports horse. Equine Vet. J., v.22, p.186-193, 1990.

JORDÃO, L.P.; CAMARGO, M.X. Estudo sobre algumas medidas do cavalo Mangalarga criado na Coudelaria Paulista. Bol. Ind. Anim., v.11, p.81-95, 1950.

LAGE, M.C.G.R. Caracterização morfométrica dos aprumos e do padrão de deslocamento de equinos da raça Mangalarga Marchador e suas associações com a qualidade da marcha. 2004. 114p. Tese (Doutorado) - Escola de Veterinária, Universidade Federal de Minas Gerais, Belo Horizonte.

LAZZERI, L. Lições de podologia equina. Belo Horizonte: EVUFMG, 1992. 224p.

LEACH, D.H.S.; ORMROD, K.; CLAYTON, H.M. Standardised terminology for the description and analysis of equine locomotion. Equine Vet. J., v.16, p.522-528, 1984.

MOLINA, A.; VALERA, M.; SANTOS, R. et al. Genetic parameters of morphofunctional traits in Andalusian horse. Livest. Prod. Sci., v.60, p.295-303. 1999.

MOTA, M.D.S. Conformação e desempenho de equinos. $1999 . \quad$ Disponível em: http://www.terravista.ciclone.com.br/ipanema/11 31/palescolin.htm. Acessado em 10 set. 2007.

NASCIMENTO, J.F. Mangalarga Marchador: tratado morfofuncional. Belo Horizonte: Associação Brasileira dos Criadores do Cavalo Mangalarga Marchador, 1999. 577p.

PROCÓPIO, A.M.; BERGMANN, J.A.G.; MENZEL, H.J. et al. Curvas ângulo-tempo das articulações dos equinos marchadores. Arq. Bras. Med. Vet. Zootec., v.59, p.41-48, 2007.

SAASTAMOINEN, M.T. Heritabililities for body size and growth rate and phenotypic correlations among measurements in young horses. Acta Agri. Scand., v.40, p.377-386, 1990.

SIERRA, G.F.; CÓRDOBA, V.M.; ALCALÁ, A.M. La valoración morfológica lineal en el caballo de Pura Raza Española. Av. Alim. Mejora Anim., v.38, p.7-10, 1998.

ZAMBORLINI, L.C. Estudo Genético Quantitativo das Medidas Lineares da Raça Mangalarga Marchador. 1996. 39f. Tese (Doutorado) - Escola de Veterinária, Universidade Federal de Minas Gerais, Belo Horizonte.

ZAMBORLINI, L.C.; BERGMANN, J.A.G.; PEREIRA, C.S. Estudo genético quantitativo das medidas lineares de equinos da raça Mangalarga Marchador - I. Estimativas dos fatores de ambiente e parâmetros genéticos. Rev. Bras. Cien. Vet., v.3, p.33-37, 1996. 